\title{
Noise-Induced Origin of the Fundamental Scalar Field
}

\author{
B. I. Lev, A. G. Zagorodny \\ Bogolyubov Institute for Theoretical Physics, NAS of Ukraine, Kyiv, Ukraine \\ Email: bohdan.lev@gmail.com
}

How to cite this paper: Lev, B.I. and $\mathrm{Za}$ gorodny, A.G. (2020) Noise-Induced Origin of the Fundamental Scalar Field. Journal of Modern Physics, 11, 502-508. https://doi.org/10.4236/jmp.2020.114032

Received: February 14, 2020

Accepted: March 27, 2020

Published: March 30, 2020

Copyright $\odot 2020$ by author(s) and Scientific Research Publishing Inc. This work is licensed under the Creative Commons Attribution International License (CC BY 4.0).

http://creativecommons.org/licenses/by/4.0/

\begin{abstract}
The physical nature of the fundamental scalar field generation and hence the origination of the Universe is a matter of the discussions for many years. We propose to use the statistical approach to the description of the steady states of the quasi stationary systems with the elements of the quantum field theory methods as a basis to explain the appearance of the cosmological scalar field. Particularly, we apply two fundamental principles, i.e., the H-theorem and least-energy principle to show principal possibility of the scalar field origination. Along with the basic statement that in the presence of the fundamental scalar field, the energy of the vacuum ground state is lower than the ground state energy of the vacuum with no scalar field (primary vacuum), and with regard to the nonlinear interaction of fluctuating physical fields with the scalar field, these principles are employed to reveal probable phase transitions that may be associated with origin and further evolution of the Universe. Thus, we propose the possible physical justification of the spontaneous cosmological scalar field generation.
\end{abstract}

\section{Keywords}

Cosmology, Noise, Fundamental Scalar Field

\section{Introduction}

Modern notions (rather hypotheses) on the reason for the formation of the Universe imply an instability of some (hypothetical) scalar fields associated with the quantum nature of the matter [1]. The reasons and physical mechanism of the appearance of this field, and hence of the origination of the Universe, remain for many years a question open for a discussion. We propose one more approach similar to that proposed in [2] [3] [4] —-to describe the origination and evolution of the Universe in terms of the first principles of statistical mechanics and quantum 
theory. Our assumptions are given in what follows.

In the case of spontaneous generation of the scalar field in vacuum, the ground-state energy of the "new" vacuum (i.e. the initial vacuum plus the scalar field) for the fields of other nature should be lower than the ground state energy of the "initial" vacuum. Moreover, the self-consistent interaction of the scalar field with fluctuations of any other field provides energy conservation for the new state of the system. Calculations of the partition function for this system reveal a probability of a phase transition from the state with zero scalar field to the state with finite spontaneously generated scalar field.

\section{Statistical Distribution in Energy Space}

According to Gibbs [5], we can always pass from the description in terms of phase variables to the description in terms of energy. Hence, we may treat the entropy as a function of energy and employ the quasi-equilibrium Gibbs distribution to calculate the partition function. We can begin with the statistical description of the Universe based on the Gibbs distribution in the energy representation [5]. The canonical Gibbs distribution in the phase space is given by

$$
\rho(q, p) \mathrm{d} \Gamma=\exp \left\{\frac{F-H(q, p)}{\Theta}\right\} \mathrm{d} \Gamma
$$

where $H(q, p)=E$ is the Hamiltonian on the hypersurface of the constant energy $E, \mathrm{~d} \Gamma=\prod_{i} \mathrm{~d} q_{i} \mathrm{~d} p_{i}$ is an element of the phase space, $\Theta=k T, T$ is the temperature, and $F$ is the free energy that can be found from the normalization condition $\int \exp \left(\frac{F-H(q, p)}{\Theta}\right) \mathrm{d} \Gamma=1$. The phase space is known [5] to be determined by the energy of the system and by external parameters. We introduce the quantity $\Sigma=\ln \frac{\mathrm{d} \Gamma}{\mathrm{d} E}$. Then we can pass to the distribution in the energy space

$$
\rho(E) \mathrm{d} E=C \exp \left\{\frac{F-E}{\Theta}+\Sigma(E)\right\} \mathrm{d} E .
$$

The normalization condition yields $\int c \exp \left(\frac{F-E}{\Theta}+\Sigma(E)\right) \mathrm{d} E=1$. In order to select the states with dominant contributions in the partition function, we employ the condition for the temperature given by $\frac{\mathrm{d} \Sigma}{\mathrm{d} E}=\frac{1}{\Theta}$.

We assume that the relation between the changes of the value of the phase space from the energy $E$ is known. In terms of this definition and within the context of fundamental principles of statistical mechanics [6] that $\Sigma=\ln \frac{\mathrm{d} \Gamma}{\mathrm{d} E}=S$ reproduces the entropy of the system bearing in mind that the temperature describes dependence of entropy only on energy but not on the other thermodynamic functions. It also follows that integration over energy in the continual sense yields an expression for the partition function. It is obvious that the extreme contribution in the partition function is associated with the states for 
which $F=E-\theta S$ and that for any deviations from the latter condition the contribution in the partition function is negligibly small similarly to the contribution of quantum corrections to the classical trajectories [7] [8].

The Universe is non-equilibrium from origination, so in order to describe its evolution we introduce an additional intrinsic parameter "time". We assume that both the statistical distribution and the evolution of the Universe can be described in terms of the distribution function that depends only on energy. An example of how this idea is applied to describe the properties of the statistical distribution is given in [7] [8]. It seems quite natural to suggest the evolution of the system in the energy space to be analogous to the Brownian motion in such a space. This raises the question which system can serve for the Universe as a thermostat. It is reasonable to suggest that such thermostat is the vacuum with fluctuations of all physical fields that interact with the fundamental scalar field and thus influence even the ground state of the vacuum. On the other hand, this suggestion opens the possibility to describe the Universe evolution in the energy space by the appropriate distribution function governed by the Fokker-Planck equations with nonlinear energy dependence of the diffusion and dissipation coefficients associated with relevant nonlinear Langevine equations [7] [8]. Just this assumption shows the way to describe the evolution of the Universe both before and after origin.

Now let us apply the above speculations to the description of the Universe. First, we suggest that the vacuum ground state possesses energy. We also assume that fluctuations of all fields existing in vacuum can occur and thus we can write the equation of state for the vacuum. The thermodynamic relations yield the pressure given by $P=-\Theta \frac{\mathrm{d} S}{\mathrm{~d} V}$ where $V$ is the volume. For pure vacuum, we have $P=-\Theta \frac{\mathrm{d} S}{\mathrm{~d} E_{v}} \frac{\mathrm{d} E_{v}}{\mathrm{~d} V}=-\frac{\mathrm{d} E_{v}}{\mathrm{~d} V}=-\rho_{v}$ under the assumption that energy with density $\rho_{v}$ is additive, i.e., $E_{v}=\rho_{v} V$ and constant entropy. Obtained equation reproduces the known equation of state for the vacuum. In order to describe its evolution we introduce an additional intrinsic parameter, "time", and write $\dot{S}=\frac{\mathrm{d} S}{\mathrm{~d} E} \dot{E}=\frac{1}{\Theta} \dot{E}$. The latter equation implies that time changes of the entropy are related to the time changes of energy. Inasmuch as $\dot{S}>0$, relaxation to the equilibrium state occurs for $\dot{E}>0$, i.e., energy growth is accompanied by the increase of entropy. Thermodynamics regards heat as energy distributed between the degrees of freedom that are not macroscopic observable. Hence we suggest that under the change of the vacuum state the heat $\mathrm{d} Q=\Theta \mathrm{d} S$ varies as $\frac{\mathrm{d} Q}{\mathrm{~d} t}=\frac{\mathrm{d} E}{\mathrm{~d} t}$ which in turn implies that heating can occur only under the relaxation towards equilibrium state.

\section{Origin of Classical Fundamental Scalar Field}

The above consideration and relations are well known. Now we employ them to 
propose one more possible explanation of the origin of fundamental scalar field. We begin with the assumption that the phase transition from the "initial" vacuum to the new vacuum state is accompanied by the appearance of new scalar field. This means that the presence of the scalar field makes the "new" vacuum different from the "primary" vacuum for any field that may exist. The scalar field decreases the energy of the "new" vacuum with respect to the energy of the "primary" vacuum. Hence, the ground-state energy of the "new" vacuum is given by

$$
E=E_{v}-\frac{\mu_{0}^{2}}{2} \varphi^{2}
$$

Here the second term is the scalar field energy; the coefficient $\mu_{0}^{2}$ describes the coupling of the new field and the "primary" vacuum, i.e., the self-consistent interaction of the new field with the fluctuations of all other fields that can exist in the "primary" vacuum. Notice, that the coupling coefficient is now positive so there is no need to use the explanations accepted in the standard approach. The contribution of the above interaction to the partition function (2) is given by

$$
Z \sim \int D \varphi \int D \xi \exp \frac{1}{\Theta}\left\{-E_{v}+\frac{1}{2} \mu^{2} \varphi^{2}-\frac{1}{2} \xi \varphi^{2}-\frac{\xi^{2}}{\sigma^{2}}\right\}
$$

where the coupling coefficient is presented in terms of its average value plus the fluctuation caused by the nonlinear coupling of the scalar field with a fluctuation field of other nature. We also assume that the mean-square value of the fluctuation is equal to $\mu_{0}^{2}=\mu^{2}+\xi . \sigma^{2}$ is dispersion of the couples coefficient fluctuations. Integration over fluctuation fields yields

$$
Z \sim \int D \varphi \exp \frac{1}{\Theta}\left\{-E_{v}+\frac{1}{2} \mu^{2} \varphi^{2}-\frac{\sigma^{2} \varphi^{4}}{4}\right\}
$$

This means that we have a system with the effective energy (averaged over the fluctuations of the other field coupled with the scalar field) given by

$$
E=E_{v}-\frac{1}{2} \mu^{2} \varphi^{2}+\frac{\sigma^{2} \varphi^{4}}{4}
$$

where the last two-term is the well-known expression for the energy of the fundamental scalar field $V(\varphi)=-\frac{1}{2} \mu^{2} \varphi^{2}+\frac{\sigma^{2} \varphi^{4}}{4}$. The total effective energy of the "new" vacuum with the fundamental scalar field is given by

$$
E=E_{v}-\frac{\mu^{4}}{4 \sigma^{2}}+\frac{\sigma^{2}}{4}\left(\varphi^{2}-\frac{\mu^{2}}{\sigma^{2}}\right)^{2}
$$

In the case of no scalar field $\varphi=0, E=E_{v}$ while for $\varphi^{2}=\frac{\mu^{2}}{\sigma^{2}}$ the expression for the effective ground state energy of the "new" vacuum reduces to $E=E_{v}-\frac{\mu^{4}}{4 \sigma^{2}}$. As follows from last relation, the energy of the "new" vacuum is lower than the energy of the primary vacuum and can vanish for $E_{v}=\frac{\mu^{4}}{4 \sigma^{2}}$. 
This relation can be applied to estimate the maximum dispersion of the field fluctuations. If $\sigma^{2}$ tends to infinity, then the energy of the new state tends to the initial energy of the ground state. Thus, we come to the standard form of the energy of the fundamental scalar field, but with different behavior of the energy of vacuum at the presence of the scalar field. The coefficient of non-linearity in the potential energy is determined by the coupling of the fundamental scalar field with the fluctuations of the field of different nature. This means that there could be a new scenario of the Universe formation. In this scenario, the energy of pure vacuum does not contribute to the energy-momentum tensor and thus we cannot introduce dynamic presentation (and geometry) for such state. Only, if the fundamental scalar field appears and the matter is originated we can tell about the geometry. In this sense, the potential of scalar field determines the vacuum state of the Universe.

\section{Conclusions}

Standard cosmological models involve a scenario of the Universe nucleation and expansion based on a scalar field which is of fundamental importance for the unified theories of weak, strong, and electromagnetic interactions with spontaneous symmetry breaking [1]. A theory of new-phase bubble nucleation and expansion was proposed in Ref. [1]. Various cosmological models describe tunneling through the potential barrier in terms of the potentials $V(\varphi)$ of arbitrary forms. Here we propose a modification of the standard cosmological model. As was mentioned above, we assume that the fundamental scalar field interacts with possible fluctuations of fields of the other nature. To proceed further and to calculate the size of the bubble, we have to violate the equivalence of the local minima. Attempts have been made [1] to obtain non-linearity of such type associated with the fluctuations of the medium and produced by the interaction with the fields of different nature [9] [10]. Similar ideas are used in the description of phase transitions in condensed media, e.g., liquid crystals, superconductors etc. [11]. In order to explain the fact that the transition in such systems is the firstorder one, the physical mechanism has been reduced to the interaction of the scalar order parameter with the vector field that includes information on possible fluctuations in the system [11] [12]. This means that the contribution of all existing configurations of such fields results in the additional part in the potential energy which proportional $\varphi^{3}$ in turn violates the equivalence of the local minima and opens the possibility to determine the bubble size of new phase with nonzero fundamental scalar field [12]. Thus the "condensing" value of fluctuations of the field that is "external" with respect to the scalar field completely determines both the mean critical size of the new phase bubble and the probability of its formation. In this case characteristics of such formation have no free parameters other than fluctuation dispersion.

As mentioned above, only the fundamental scalar field and its symmetry breaking generate the matter, and equilibrium distribution of this matter deter- 
mines the geometry [13]. Obviously, the size of the bubble of the new phase is a finite one that is in agreement with the observations. And here we notice that in the Einstein equation the energy of primary vacuum is not present and the dynamics of the Universe are determined only by the potential energy of the fundamental scalar field which produces the matter. The distribution of matter in its turn determines the geometry. For our Universe, the vacuum is different from the primary one and its state depends on the fundamental scalar field which determines all possible processes. So, the dispersion of the fluctuations fully determines the conditions of new phase bubble formation.

Thus, the assumption of the vacuum ground state energy decrease for all physical fields due to the presence of the fundamental scalar field makes it possible to reveal the probability of a phase transition caused by the spontaneous generation of the field, i.e. the phase transition from vacuum with zero scalar field to the "new" vacuum with the spontaneously generated field. Combining this assumption with the idea that the Universe interacts with the fluctuations of various physical fields in vacuum we can get a consistent picture of the Universe origination and evolution. The decrease of the initial ground state energy does not contradict the $\mathrm{H}$-theorem, because the distribution functions describing the evolution governed by the Fokker-Planck equation are known to satisfy it [14] [15].

\section{Acknowledgements}

This work was supported by the Target Program of Fundamental Research of the Department of Physics and Astronomy of the National Academy of Sciences of Ukraine (N0120U100857).

\section{Conflicts of Interest}

The authors declare no conflicts of interest regarding the publication of this paper.

\section{References}

[1] Linde, A.D. (1990) Particle Physics and Inflationary Cosmology. CRC Press, London, New-York, Washington D.C.

https://doi.org/10.1016/B978-0-12-450145-4.50004-9

[2] Fomin, P.I. and Fomina, A.P. (2015) Dark Energy and Dark Matter in the Universe. In: Shulga, V., Ed., Kyiv, Akademperiodik, Vol. 3, 15.

[3] Fomin, P.I. (1976) Physics of Elementary Particles and Atomic Nuclei, 7, 682.

[4] Boyle, L., Finn, K. and Turok, N. (2018) Physical Review Letters, 121, Article ID: 251301. https://doi.org/10.1103/PhysRevLett.121.251301

[5] Gibbs, J.W. (1902) Elementary Principles in Statistical Mechanics, Developed with Especial Reference to the Rational Foundation of Thermodynamics. Charles Scriener's Sons, New York; Edward Arnold, London. https://doi.org/10.5962/bhl.title.32624

[6] Landau, L.D. (1973) Statistical Mechanics. Nauka, Moskwa. 
[7] Lev, B.I. (2013) The European Physical Journal Special Topics, 216, 37-48. https://doi.org/10.1140/epjst/e2013-01727-1

[8] Lev, B.I. and Kiselev, A.D. (2010) Physical Review E, 82, Article ID: 031101. https://doi.org/10.1103/PhysRevE.82.031101

[9] Horsthemke, W. and Lefever, R. (1984) Noise-Induced Transition. In: Theory, Applications in Physics, Chemistry and Biology, Springer-Verlag, New-York.

[10] Lev, B.I. (2018) Journal of Modern Physics, 9, 2223-2232. https://doi.org/10.4236/jmp.2018.912140

[11] Chaikin, P.M. and Luensky, T.C. (1995) Principles of Comdensed Matter Physics. Cambridge University Press, Cambridge.

[12] Lev, B. and Yokoyama, H. (2003) International Journal of Modern Physics B, 17, 4913-4933. https://doi.org/10.1142/S021797920302274X

[13] Rebesh, A.P. and Lev, B.I. (2017) Physics Letter A, 381, 2538-2543. https://doi.org/10.1016/j.physleta.2017.05.051

[14] Balesku, R. (1978) Equilibrium and Non-Equilibrium Statistical Mechanics. J. Wiley and Sons, New York.

[15] Nelson, E. (2001) Dynamical Theories of Brownian Motion. 2nd Edition. Princeton University Press, Princeton, 114. 